\title{
Diffractive Open Charm Production at $\mathrm{H} 1$
}

\author{
Paul Thompson ${ }^{1}$ \\ 1- School of Physics - University of Birmingham \\ BIRMINGHAM B15 2TT - UK
}

\begin{abstract}
Measurements are presented [1] of diffractive open charm production at HERA where two analysis techniques are used for the cross section measurements. In the first, the charm quark is tagged by the reconstruction of a $D^{* \pm}(2010)$ meson. This technique is used in deep-inelastic scattering (DIS) and photoproduction $(\gamma p)$. In the second, a method based on the displacement of tracks from the primary vertex is used to measure the open charm contribution to the inclusive diffractive cross section in DIS. The measurements are compared with next-to-leading order QCD predictions based on diffractive parton density functions previously obtained from a QCD analysis of the inclusive diffractive cross section at $\mathrm{H} 1$.
\end{abstract}

\section{Introduction}

In quantum chromodynamics (QCD), the theory of strong interactions, the hard scattering collinear factorization theorem [2] predicts that the cross section for diffractive deep-inelastic ep scattering (DIS) factorizes into a set of universal diffractive parton density functions (DPDFs) of the proton and process-dependent hard scattering coefficients. Next-to-leading order (NLO) DPDFs have been determined by QCD fits to the measured cross sections of inclusive diffractive scattering at HERA [3] within the factorizable Pomeron model [4] and using the DGLAP evolution equations. The DPDFs have been found to be dominated by gluons, which carry $\approx 70 \%$ of the momentum of the diffractive exchange.

In the collinear factorization approach diffractive open charm production at HERA is expected mainly to proceed via boson gluon fusion (BGF). Thus it is directly sensitive to the gluon content of the diffractive exchange, which is only determined indirectly and for low momentum fractions $z_{\mathbb{P}}$ of the gluon in inclusive diffractive scattering via scaling violations [3]. In the BGF process a charm quark anti-quark pair $(c \bar{c})$ is produced of which one quark couples to the photon with virtuality $Q^{2}$ and the other to a gluon that emerges from the diffractive exchange.

In an alternative theoretical approach DPDFs are not introduced and diffractive scattering is explicitly modeled by the perturbative exchange of a colorless gluon state (two gluons or a gluon ladder). Formulated in the proton rest frame the "two-gluon" state of the proton can couple directly to the $c \bar{c}$ pair $\left(\gamma^{*} p \rightarrow c \bar{c} p\right)$ or to a $c \bar{c} g$ color dipole fluctuation of the photon $\left(\gamma^{*} p \rightarrow c \bar{c} g p\right)$ [5]. A model combining the perturbative two-gluon approach with the collinear factorization scheme, which has also been used to fit the HERA diffractive DIS cross sections, is given in [6].

This article is based on the recent paper on diffractive open charm production from H1 appearing in [7]. Two methods to identify charm production are presented. In the first method the charm quark is tagged by the reconstruction of $D^{*}$ mesons. The measurement is performed in DIS and, due to the high selectivity of the trigger, extended to photoproduction $(\gamma p)$. The second method, which was used to measure the total inclusive charm and beauty cross sections in DIS [8, 9], is used for the first time in diffractive DIS. In this method, referred to in the following as the 'displaced track analysis', the charm quark is identified 
by the reconstruction of tracks, which are displaced from the interaction vertex, that arise due to long lived charmed hadrons. This method is used in a kinematic region with high acceptance for the decay products of charmed hadrons within the silicon vertex detector of $\mathrm{H} 1$, which is used in the reconstruction of these tracks. With this method it is thus possible to measure the total open charm contribution to the diffractive cross section with small extrapolations from QCD calculations.

\section{Results}

Diffractive $D^{* \pm}$ production in DIS is studied in the kinematic range of $2<Q^{2}<100 \mathrm{GeV}^{2}$, $0.05<y<0.7, x_{\mathbb{P}}<0.04, M_{Y}<1.6 \mathrm{GeV},|t|<1 \mathrm{GeV}^{2}, p_{t}\left(D^{*}\right)>2 \mathrm{GeV}$, and $\left|\eta\left(D^{*}\right)\right|<$ 1.5. In $\gamma p$ diffractive $D^{* \pm}$ production is studied in the kinematic range of $Q^{2}<0.01 \mathrm{GeV}^{2}$, $0.3<y<0.65, x_{\mathbb{P}}<0.04, M_{Y}<1.6 \mathrm{GeV},|t|<1 \mathrm{GeV}^{2}, p_{t}\left(D^{*}\right)>2 \mathrm{GeV}$ and $\left|\eta\left(D^{*}\right)\right|<1.5$. A good agreement between the data cross sections, integrated over the kinematic ranges given above, and the NLO QCD calculations is observed.

The $D^{*}$ meson cross section in DIS is also measured differentially as a function of various kinematic variables. For example, in Figure 1 the cross section as a function of $x_{\mathbb{P}}$ and an estimate of the invariant mass of the $c \bar{c}$ pair emerging from the hard scattering process $z_{\mathbb{P}}^{o b s}$ is shown. The data are compared in the figure with the predictions of NLO QCD calculations
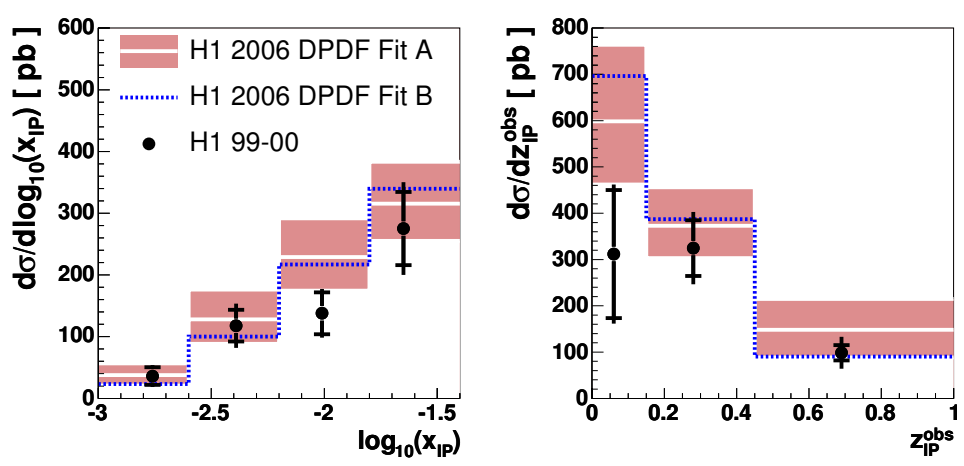

Figure 1: The diffractive $D^{*}$ cross section in DIS shown as a function of $x_{\mathbb{P}}$ and $z_{\mathbb{P}}^{o b s}$. based on two ( Fit A and Fit B) alternative sets of diffractive parton density functions from $\mathrm{H} 1$ [3], which both provide a good description of the inclusive diffractive DIS data. The differences in the predictions for the two DPDFs are moderate, with $z_{\mathbb{P}}^{o b s}$ showing the greatest sensitivity. However, within the present experimental errors and theoretical uncertainties these differences cannot be resolved.

In $\gamma p$ the $D^{*}$ cross section is also shown differentially as a function of the diffractive kinematic variables $x_{\mathbb{P}}$ and $z_{\mathbb{P}}^{o b s}$ in Figure 2. The data are well described by the theoretical predictions within the larger experimental errors for $\gamma p$. As in DIS the largest sensitivity to the different parameterizations of the gluon is evident in the $z_{\mathbb{P}}^{o b s}$ distribution.

The good agreement of the NLO QCD predictions with the measured cross sections observed in DIS and $\gamma p$, both in shape and normalization, supports the assumption that QCD factorization is applicable in both kinematic regimes. A quantity, which is less sensitive to the input of diffractive parton density functions and theoretical uncertainties is defined by $R_{\mathrm{DIS}}^{\gamma p}=\frac{\left(\sigma^{\text {meas }} / \sigma^{\text {theo }}\right)_{\gamma p}}{\left(\sigma^{\text {meas }} / \sigma^{\text {theo }}\right)_{\text {DIS }}}$ where $\sigma^{\text {meas }}$ and $\sigma^{\text {theo }}$ denote the measured and the predicted 
integrated cross section for $D^{*}$ production. The ratio $R_{\mathrm{DIS}}^{\gamma p}$ is found to be $1.15 \pm 0.40$ (stat.) \pm 0.09 (syst.). The theoretical uncertainty on $R_{\mathrm{DIS}}^{\gamma p}$ is $\pm 7 \%$. The measurement of $R_{\mathrm{DIS}}^{\gamma p}$ shows no evidence for a suppression of the $\gamma p$ component although the statistical error of the measurement is large.
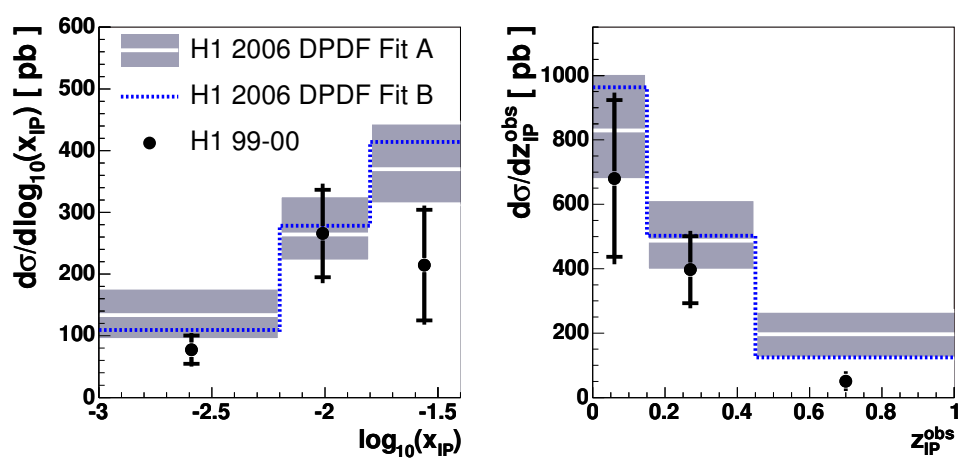

Figure 2: The diffractive $D^{*}$ cross section in $\gamma p$ shown as a function of $x_{\mathbb{P}}$ and $z_{\mathbb{P}}^{o b s}$.

An additional comparison of both the NLO QCD calculations and of the prediction from the perturbative two gluon calculation of BJKLW [5] with differential cross sections has been made in the range of validity of the model $\left(x_{\mathbb{P}}<0.01\right)$. Good agreement between the data and both the model of BJLKW and the NLO QCD calculations is observed in this kinematic range. For the two gluon calculation the $\gamma^{*} p \rightarrow c \bar{c} g p$ contribution is seen to dominate with the $\gamma^{*} p \rightarrow c \bar{c} p$ process contributing only at high values of $z_{\mathbb{P}}^{o b s}$.
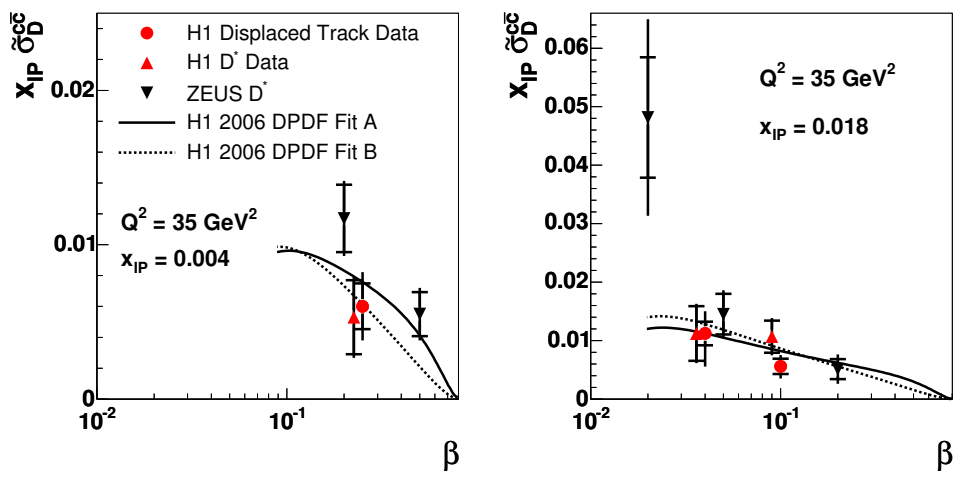

Figure 3: The reduced cross section $x_{\mathbb{P}} \tilde{\sigma}_{D}^{c \bar{c}}$ shown as a function of $\beta$ for two different values of $x_{\mathbb{P}}$.

small for the data points presented.

The measurements of $x_{\mathbb{P}} \tilde{\sigma}_{D}^{c \bar{c}}$ obtained from the displaced track method are shown in Figure 3 as a function of $\beta$ for fixed values of $Q^{2}$ and $x_{\mathbb{P}}$. The measured points of $x_{\mathbb{P}} \tilde{\sigma}_{D}^{c \bar{c}}$ are compared with the results extracted from the $D^{*}$ meson analysis. For this purpose the $D^{*}$ cross section is measured in the same kinematic ranges as for the displaced track method. These measurements in the visible $D^{*}$ kinematic range $p_{t}\left(D^{*}\right)>2 \mathrm{GeV}$ and $\left|\eta\left(D^{*}\right)\right|<1.5$
The measurements of the diffractive charm DIS cross sections obtained from the displaced track method are used to measure the diffractive charm reduced cross section. The reduced cross section is approximately equal to the charm contribution $F_{2}^{D(3) c \bar{c}}$ to the diffractive structure function $F_{2}^{D(3)}$. The difference is due to the contribution from the longitudinal diffractive charm cross section, which is expected to be 
are extrapolated with the NLO calculation program HVQDIS to the full $D^{*}$ kinematic phasespace in order to extract the diffractive open charm cross section. The extrapolation factors are found to be $\approx 2.5$. The $\mathrm{H} 1$ data are also compared with $D^{*}$ measurements from the ZEUS collaboration [10] which are interpolated to the same kinematic range as the H1 measurement using the NLO QCD fit and corrected with a factor of 1.23 to account for the difference in the measured range of $M_{Y}$ of the experiments. The measurements for $x_{\mathbb{P}} \tilde{\sigma}_{D}^{c \bar{c}}$ from the displaced track analysis and the $D^{*}$ extraction methods from both $\mathrm{H} 1$ and ZEUS are in good agreement. A comparison with the predictions of the NLO DPDFs shows a good description of the data.

The measurements have also been presented in the form of the fractional contribution of charm to the total diffractive $e p$ cross section $f_{D}^{c \bar{c}}$. In the given kinematic range the value of $f_{D}^{c \bar{c}}$ is $\approx 20 \%$ on average, which is comparable to the charm fraction in the inclusive cross section at low values of Bjorken $x$ for similar values of $Q^{2}$ [9]. The inclusive measurements have also been compared with the predictions of the MRW model [6]. A good description of the data is observed supporting the validity of the DPDFs extracted in this model.

\section{Summary}

Measurements have been presented of the diffractive charm cross section in DIS and $\gamma p$ using two independent methods of charm reconstruction. A comparison with QCD calculations in NLO based on DPDFs obtained from inclusive diffractive scattering at $\mathrm{H} 1$ is in good agreement with the measurement in both kinematic regimes. No evidence is observed for a suppression in $\gamma p$. At low $M_{X}$ the data are found to be also well described by models based on two gluon exchange and diffractive parton densities.

\section{Bibliography}

\section{References}

[1] Slides:

http://indico. cern.ch/contributionDisplay . py? contribId=82\&sessionId=13\&conf Id $=9499$

[2] J. C. Collins, Phys. Rev. D 57 (1998) 3051 [Erratum-ibid. D 61 (2000) 019902] [hep-ph/9709499].

[3] H1 Collaboration, "Measurement and QCD analysis of the diffractive deep-inelastic scattering cross section at HERA", DESY 06-049, accepted by Eur. Phys. J. C [hep-ex/0606004].

[4] G. Ingelman, and P. Schlein, Phys. Lett. B 152 (1985) 256;

A. Donnachie and P. Landshoff, Phys. Lett. B 191 (1987) 309 [Erratum-ibid. B 198 (1987) 590].

[5] J. Bartels, H. Lotter, M. Wüsthoff, Phys. Lett. B 379 (1996) 239 [Erratum-ibid. B 382 (1996) 449] [hep-ph/9602363];

J. Bartels, C. Ewerz, H. Lotter, M. Wüsthoff, Phys. Lett. B 386 (1996) 389 [hep-ph/9605356];

J. Bartels, H. Jung, M. Wüsthoff, Eur. Phys. J. C 11 (1999) 111 [hep-ph/9903265];

J. Bartels, H. Jung, A. Kyrieleis, Eur. Phys. J. C 24 (2002) 555 [hep-ph/0204269].

[6] A. D. Martin, M. G. Ryskin, G. Watt, Eur. Phys. J. C 44 (2005) 69 [hep-ph/0504132];

[7] A. Aktas et al., [H1 Collaboration], Eur. Phys. J. C 50 (2007) 1 [hep-ex/0610076].

[8] A. Aktas et al., [H1 Collaboration], Eur. Phys. J. C 40 (2005) 349 [hep-ex/0411046].

[9] A. Aktas et al., [H1 Collaboration], Eur. Phys. J. C 45 (2006) 23 [hep-ex/0507081].

[10] S. Chekanov et al., [ZEUS Collaboration], Nucl. Phys. B 672 (2003) 3 [hep-ex/0307068]. 